\title{
Proton Beam Therapy and Continuous Intra-Arterial Chemotherapy for Polymorphous Low-Grade Adenocarcinoma in the Hard Palate
}

\author{
Chiyoko Makita $^{a}$ Tatsuya Nakamura ${ }^{a}$ Kanako Takayama ${ }^{a}$ \\ Akinori Takada $^{a}$ Nobukazu Fuwa ${ }^{c}$ Hideo Sakuma $^{b}$ \\ ${ }^{a}$ Department of Radiation Oncology, Southern Tohoku Proton Therapy Center, and \\ ${ }^{b}$ Department of Pathology, Southern Tohoku Hospital, Fukushima, and ${ }^{\mathrm{c} H y o g o}$ Ion Beam \\ Medical Center, Tatsuno, Japan
}

\section{Key Words}

Polymorphous low-grade adenocarcinoma - Proton beam therapy · Continuous intra-arterial chemotherapy

\begin{abstract}
Polymorphous low-grade adenocarcinoma is a rare malignant tumor that mainly occurs in salivary glands, particularly in the hard palate. To date, surgery has been the most commonly used treatment method with the aim of achieving negative margins of resection. Here, we report a case of a 51-year-old woman who presented with a painless growing mass in her oral cavity with Rouviere's lymph node metastasis. Laboratory data showed severe anemia because of bleeding from the tumor. We inserted two catheters into the bilateral external carotid arteries via the superficial temporal arteries, and began continuous intra-arterial infusion with cisplatin and a cisplatin-neutralizing agent. The bleeding stopped after the first infusion, and we continued with weekly arterial cisplatin infusion and proton beam therapy (70.4 GyE in 32 fractions). A decrease in tumor volume has been maintained for 2 years, with no evidence of new metastasis.
\end{abstract}




\section{Introduction}

Polymorphous low-grade adenocarcinoma (PLGA) is a rare tumor that mainly occurs in minor salivary glands, particularly in the hard palate. PLGA can be summarized as a cytologically uniform and morphologically diverse malignancy with a low metastatic potential. Treatment for the primary lesion involves surgical resection with a safety margin, which is sometimes difficult considering the surrounding essential structures; however, the tumor has the potential to be fatal. In 2009, we began proton beam therapy in combination with continuous intra-arterial chemotherapy in patients with advanced oral/maxillary cancer in whom surgery was impossible or who refused surgery. Here, we report the effectiveness of proton beam therapy and continuous intra-arterial chemotherapy in locally aggressive PLGA in the hard palate.

\section{Case Presentation}

A 51-year-old woman presented with a painless tumor in her palate, which had gradually developed a few years before presentation. She was recommended surgery at several hospitals, but refused treatment. The size of the swollen area remained constant for 1 year, but the tumor developed rapidly within the last 3 months before admission. When she visited our hospital, she could not speak or chew because of the massive tumor (fig. 1) and was therefore dependent on liquid dietary supplements. She complained of slight fatigue and a weight loss of $5 \mathrm{~kg}$ over 6 months. A Physical examination on admission revealed anemia of the conjunctivae and skin, and oozing from the surface of the tumor. Her hemoglobin level was $5.3 \mathrm{~g} / \mathrm{dl}$ and histopathological examination revealed solid, tubular and cystic morphological patterns. Immunohistochemical staining was positive for cytokeratin, vimentin and S100 protein; the mindbomb homolog 1 labeling index was $10 \%$. A contrast-enhanced magnetic resonance imaging (MRI) demonstrated a tumor measuring $4.9 \times 3.3 \times 7.3 \mathrm{~cm}$, which was exophytic and well circumscribed with a connection to the hard palate. The MRI also revealed a suspected left Rouviere's lymph node metastatic lesion measuring $2.2 \times 2.1 \times$ $1.9 \mathrm{~cm}$ (fig. 2). Surgery was not an option since it seemed difficult to completely resect the lymph node due to its proximity to the internal carotid artery. After transfusion, the superficial temporal arteries (STAs) were cannulated and cisdiamminedichloroplatinum (II) (CDDP) infusion was started concurrently with proton beam therapy (fig. 3).

\section{Proton Beam Therapy}

A thermoplastic shoulder fixation system was used for patient immobilization. Serial CT (2-mm slice thickness) sections and contrast-enhanced MRI images for 3D visualization were obtained in treatment position, and they were used for treatment planning (Xio-M; CMS Japan, Tokyo, Japan; Mitsubishi Electric). The gross tumor volume, which was included in Rouviere's lymph node, was manually outlined on CT/MRI images. The clinical target volume was defined as the gross tumor volume with a 3-mm margin in all directions. The clinical target volume was expanded by $3 \mathrm{~mm}$ in all directions to create the planning target volume (PTV) for setup uncertainty, but it did not extend into the critical organs at risk such as the brain stem and spinal cord. The beam shapes were optimized using the multileaf collimator. Two portal beams were arranged in the optimal angles to avoid excess-dose exposure to the normal tissues (fig. 3). The dose was calculated based on a pencil beam algorithm. Adequate treatment parameters, including beam energy, spread-out Bragg peak width and degrader thickness, were adjusted for complete PTV coverage. The relative 
biological effectiveness of the proton beam was defined as 1.1. A total dose of 70.4 GyE in 32 fractions at the isocenter was administered to cover $90 \%$ of the PTV.

\section{Continuous Intra-Arterial Chemotherapy}

The bilateral STAs were cannulated, and tumor staining was confirmed via angiography and MRI with a low dose of contrast medium slowly infused through the catheter for arterial injection.

CDDP was administered as an intra-arterial infusion at a dose of $40 \mathrm{mg} / \mathrm{m}^{2}$ for $5 \mathrm{~h}$ once a week, for 9 courses. During arterial CDDP infusion, a CDDP-neutralizing agent, sodium thiosulfate, was administered intravenously at a dose of $8 \mathrm{~g} / \mathrm{m}^{2}$ for $8 \mathrm{~h}$, starting $1 \mathrm{~h}$ before arterial CDDP infusion.

\section{Evaluation and Follow-Up}

Follow-up examination included clinical examination at monthly intervals, contrastenhanced MRI every 3 months, and fluorodeoxyglucose positron emission tomography/CT to check distant metastasis every 6 months. Acute toxicities were assessed using the National Cancer Institute Common Terminology Criteria for Adverse Events (version 4.0).

\section{Treatment Outcome}

Hematological toxicity or neurological complications were absent; however, the patient developed grade 3 dermatitis and mucitis at the end of the treatment and received intravenous hyperalimentation for 2 weeks. One month after treatment, her clinical condition was good and she had no difficulty in the oral intake of liquid food. For 2 years after treatment, the tumor maintained a size of $3.0 \times 3.3 \times 2.7 \mathrm{~cm}$, whereas Rouviere's lymph node metastatic lesion maintained a size of $0.8 \times 0.7 \times 0.7 \mathrm{~cm}$. Therefore, a long-term followup was required. However, at present, she faces no difficulty in oral intake and speech. She has gained $4 \mathrm{~kg}$ and tumor bleeding has not been observed since treatment completion.

\section{Discussion}

PLGA is a rare tumor, especially in Japan, and it was described first in 1983 as a terminal duct carcinoma by Evans and Batsakis [1]. PLGA accounts for about 19-26\% of all malignancies of the minor salivary glands, with a predilection for females over males in a ratio of 24.6:1. The age at presentation of PLGA typically peaks in the sixth and seventh decades of life. PLGA exhibits varied histological patterns (cords, tubules, papillae, glandular structures and solid aggregates), cytological uniformity (myoepithelial or luminal duct cells) and infiltrating growth [2]. The tumor appears to occur mainly in the minor salivary glands, particularly in the hard palate, with a lower frequency in the lips, buccal mucosa and tongue. PLGA usually presents as a slowly growing enlargement, and nodal and distant metastases are rare. However, some studies have described cases of locally aggressive progression and distant spread [3].

The standard treatment for PLGA is wide local excision with clear margins. Postoperative radiation therapy is reserved for cases of positive or close surgical margins, but the role of adjuvant radiotherapy and chemotherapy remains unclear.

The overall survival of PLGA is excellent. Castle et al. [2] showed an average time to recurrence of 7.2 years. In their study with an average follow-up of 115 months, a diseasefree survival and a local control rate ( 98.2 and $97.6 \%$, respectively) were observed in their series of 164 patients. Furthermore, in their study, only 3 patients died from extensive local 
tumors after surgical excision or surgery followed by radiation/chemotherapy. Vincent et al. [4] reported a recurrence rate of $17 \%$ with a regional metastasis rate of $9 \%$. Many of the recurrences have been reported after 5 years. On the other hand, Simpson et al. [5] reported that tumors could be predisposed to potentially more malignant transformations, which are far less aggressive than an adenoid cystic carcinoma, but retain potential to be fatal in a minority of patients.

Here, we reported an inoperable, locally aggressive PLGA treated with proton beam therapy and continuous intra-arterial chemotherapy. We performed arterial CDDP infusion via the STAs for hemostasis, which was successful, and we continued with arterial infusion once a week concurrently with proton beam therapy. Proton beam has an excellent dose distribution for head and neck carcinomas and shows similar local control to the best available photon radiation with reduced treatment toxicity and minimal damage to normal tissues. In the present case, the proton beam could completely save the patient's brain, brain stem, spinal cord and bilateral parotid gland.

Radiotherapy is often combined with chemotherapy and/or surgery. It would be an interesting topic for future research to examine whether proton beam therapy could be combined with chemotherapy and/or surgery. Selective injection of cytostatic agents into the tumor-feeding artery is an attractive method to achieve higher doses of cytostatic agents in the tumors with less systemic toxicity. Previous reports have shown that a combination of radiotherapy with intra-arterial CDDP infusion is a useful approach for advanced head and neck carcinomas [6].

In conclusion, intra-arterial chemotherapy via the STAs should be considered for hemostasis of head and neck carcinomas, and proton beam therapy for the definitive treatment of unresectable, locally advanced head and neck carcinomas due to the advantages of high-dose delivery, minimal procedural complications and low systemic toxicity. To the best of our knowledge, this is the first case of PLGA treated with proton beam therapy and intra-arterial chemotherapy without any severe toxicities. The patient achieved a partial response to the therapy, but a long-term follow-up was required to assess treatment success, although a combination of proton beam therapy and intra-arterial chemotherapy offers a potential gain for the treatment of rare head and neck carcinomas.

\section{References}

1 Evans HL, Batsakis JG: Polymorphous low-grade adenocarcinoma of minor salivary glands. A study of 14 cases of a distinctive neoplasm. Cancer 1984;53:935-942.

-2 Castle JT, Thompson LD, Frommelt RA, Wenig BM, Kessler HP: Polymorphous low grade adenocarcinoma. A clinicopathologic study of 164 cases. Cancer 1999;86:207-219.

-3 Olusanya AA, Akadiri AO, Akinmoladun VI, Adeyemi BF: Polymorphous low grade adenocarcinoma: literature review and report of lower lip lesion with suspected lung metastasis. J Maxillofac Oral Surg 2011;10:60-63.

4 Vincent SD, Hammond HL, Finkelstein MW: Clinical and therapeutic features of polymorphous low-grade adenocarcinoma. Oral Surg Oral Med Oral Pathol 1994;77:41-47.

-5 Simpson RH, Pereira EM, Riberio AC, Abdulkadir A, Reis-Filho JS: Polymorphous low-grade adenocarcinoma of the salivary glands with transformation to high-grade carcinoma. Histopathology 2002;41:250-259.

6 Teymoortash A, Werner JA: Selective interaarterial cisplatin chemotherapy in treatment of advanced malignant squamous cell carcinoma of the head and neck. Cancer Therapy 2003;1:269-273. 


\section{Case Reports in Oncology}
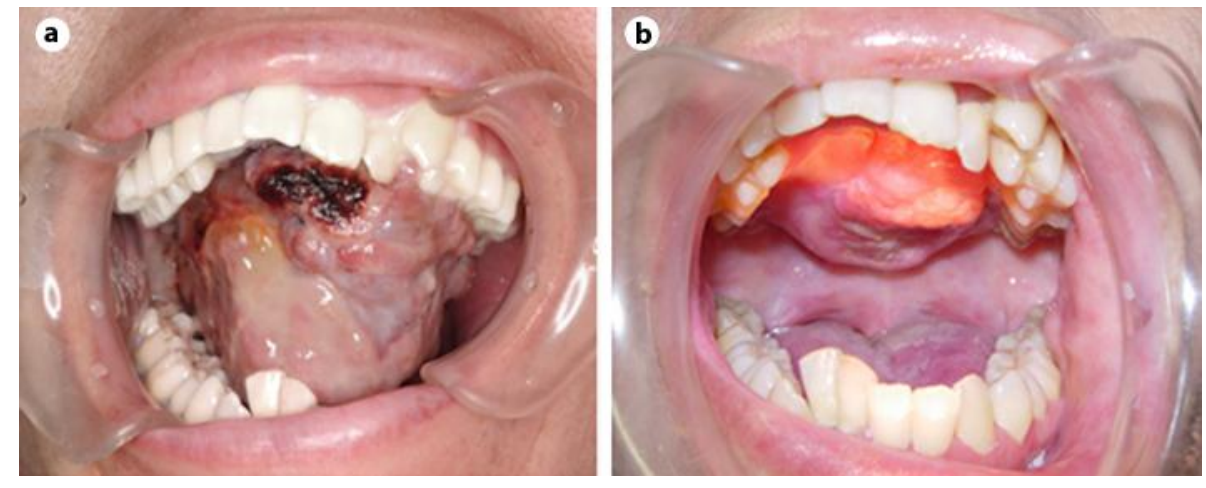

Fig. 1. The tumor in the patient's hard palate had a smooth surface. a Before treatment, bleeding from the anterior aspect of the tumor was stopped temporally by local hemostatics using the Surgicel ${ }^{\mathrm{TM}}$ Fibrillar Absorbable Hemostat (Ethicon Biosurgery, Inc., Somerville, N.J., USA). b Two years after treatment, the tumor maintained remission. 


\section{Case Reports in Oncology}
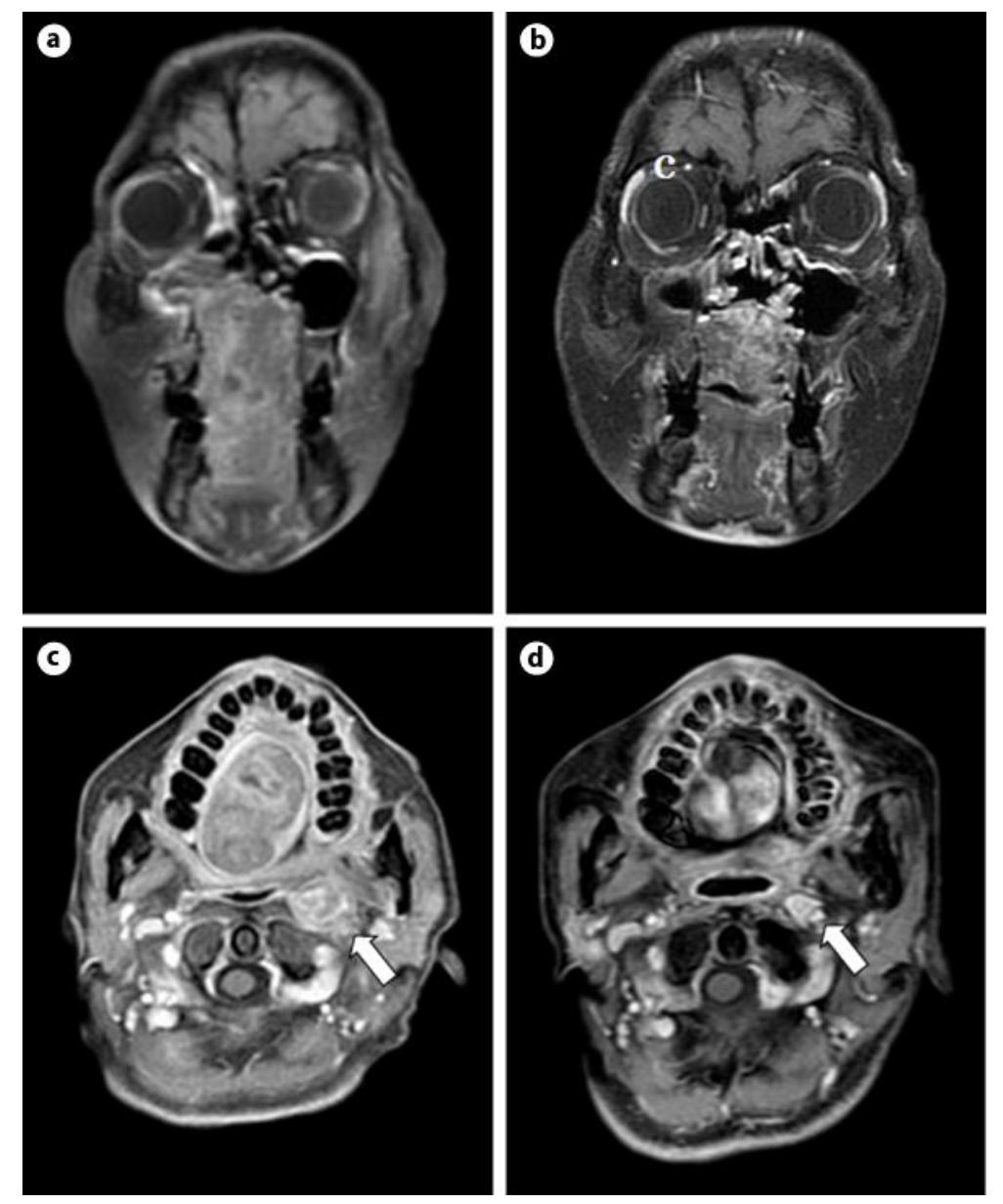

Fig. 2. Extensive PLGA originating from the hard palate with left Rouviere's lymph node metastasis (arrow). a Coronal view of the contrast-enhanced T1-weighted MRI before treatment; $\mathbf{b}$ coronal view of the contrast-enhanced T1-weighted MRI 2 years after treatment; c axial view of the contrast-enhanced T1-weighted MRI before treatment, and $\mathbf{d}$ axial view of the contrast-enhanced T1-weighted MRI 2 years after treatment.
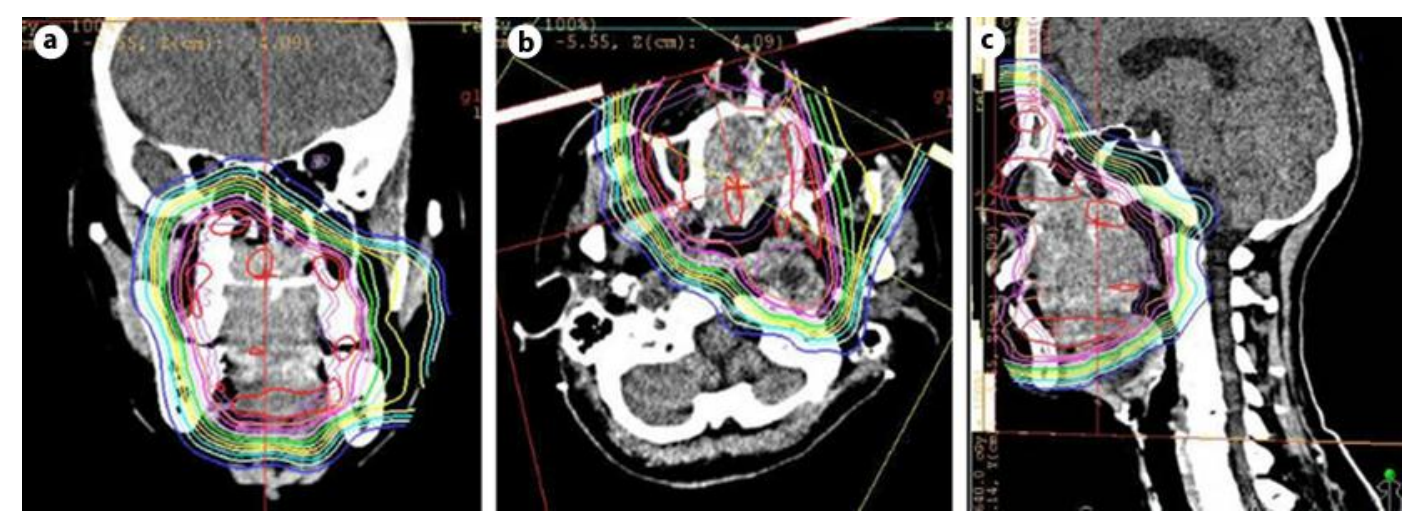

Fig. 3. Proton beam dose distribution: the tumor was treated with 70.4 GyE in 32 fractions. a Coronal view; b axial view, and c sagittal view. 